\title{
Dielectric response of laser-excited silicon at finite electron temperature
}

\author{
S.A. Sato,${ }^{1}$ Y. Shinohara, ${ }^{2}$ T. Otobe,${ }^{3}$ and K. Yabana ${ }^{4,1}$ \\ ${ }^{1}$ Graduate School of Pure and Applied Sciences, \\ University of Tsukuba, Tsukuba 305-8571, Japan \\ ${ }^{2}$ Max-Planck-Institut fur Mikrostrukturphysik, \\ Weinberg 2, D-06120 Halle, Germany \\ ${ }^{3}$ Advanced Photon Research Center, \\ JAEA, Kizugawa, Kyoto 619-0615, Japan \\ ${ }^{4}$ Center for Computational Sciences, \\ University of Tsukuba, Tsukuba 305-8577, Japan
}

\begin{abstract}
We calculate the dielectric response of excited crystalline silicon in electron thermal equilibrium by adiabatic time-dependent density functional theory (TDDFT) to model the response to irradiation by high-intensity laser pulses. The real part of the dielectric function is characterized by the strong negative behavior at low frequencies due to excited electron-hole pairs. The response agrees rather well with the numerical pump-probe calculations which simulate electronic excitations in nonequilibrium phase immediately after the laser pulse irradiation. The thermal response is also compared with the Drude model which includes electron effective mass and collision time as fitting parameters. We find that the extracted effective masses are in the range of $0.22-0.36$ and lifetimes are in the range of 1-14 fs depending on the temperature. The short Drude lifetimes show that strong damping is possible in the adiabatic TDDFT, despite the absence of explicit electron-electron collisions.
\end{abstract}




\section{INTRODUCTION}

Properties of dielectrics irradiated by high-intensity and ultra-short laser pulses have been attracting substantial interests from both fundamental and technological points of view [1 5]. We are investigating the theory of the dielectric response of materials to high fields at times shorter than the full local equilibration time. Time-domain electron dynamics simulation based on the time-dependent density functional theory (TDDFT) is quite promising for describing the earliest time. This is the subject of a companion paper, Ref. [6], where we reported numerical simulations of pump-probe experiments. This theory should describe the formation of electron-hole excitations in insulating materials and the energy deposited in them. At the next time scale, the electron-hole excitations will come to an equilibrium, allowing one to treat the system as a thermalized electron-hole plasma with fixed numbers of electrons and holes. The dielectric properties of this state are the subject of the present paper. We will discuss them in crystalline silicon as in Ref. [6]. At even later times, the atomic degrees of freedom will be thermalized as well. That complete plasma equilibrium is beyond the scope of the present work. The two-temperature model assuming much smaller time-scale of electronic equilibrium than that of phonons is well established, see Ref. [7]. We will consider the response of thermalized electrons ignoring atomic motions, keeping them at equilibrium positions in the ground state. This treatment should be reasonable at times before substantial part of the electronic excitation energies is transferred to lattice motions.

We employ a static density functional theory (DFT) at finite temperature to describe the thermalized electronic state. An extension of the DFT to nonzero electronic temperature was first considered in [8], employing the grand canonical ensemble and introducing a chemical potential for the electrons. Recent developments of finite temperature DFT include discussions on basic aspects of the theory such as the conditions for the validity of

the adiabatic connection formula [9] and applications to electrochemical reactions [10]. The finite temperature DFT has been applied to the properties of matter excited by intense and ultra-short laser pulses. For example, in Ref. [11], lattice properties of laser-excited solids were investigated using density functional perturbation theory with the Fermi-Dirac distribution for electrons. In Ref. [12], finite temperature DFT results were utilized to analyze solid aluminum excited by XUV pulses. Our implementation of finite temperature DFT will use the grand canonical ensemble for the occupation in the static solution. We then 
calculate the dielectric response in the linear response using a real-time method [14, 15].

Present thermal model calculations are different from previous numerical pump-probe simulations [6] in the population distribution of electrons. The numerical pump-probe simulations describe electronic states immediately after the laser irradiation, which are highly nonequilibrium and anisotropic. On the other hand, the present electronic thermal model describes thermalized, isotropic electronic states. In spite of these differences, we will show that many features of response in the elaborated numerical pump-probe experiments may be reproduced even at a quantitative level with the finite temperature calculation, if we compare two systems at the same number of excited electrons. We also compare with a simple Drude response embedding the free electrons in a dielectric medium [17 19$]$.

The construction of the paper is as follows. In Sec. III, we describe the theoretical framework of finite temperature model and present calculated results. In Sec. ஹ, we compare the results with the Drude model. In Sec. IV, we compare results of the finite temperature model with results of numerical pump-probe experiments. Our findings are summarized in Sec. V.

\section{ELECTRONIC THERMAL MODEL}

\section{A. Ground state}

We model the electronic state of crystalline silicon after irradiation of a high-intensity laser pulse by static DFT for a thermal ensemble of electrons. Atomic positions are kept at their equilibrium positions in the ground state, assuming that electron thermalization time is so short that atomic motions may be ignored. The Kohn-Sham equation for orbitals is given by

$$
\left\{-\frac{\hbar^{2}}{2 m_{e}} \nabla^{2}+V_{i o n}+\int d \vec{r}^{\prime} \frac{e^{2}}{\left|\vec{r}-\vec{r}^{\prime}\right|} \rho^{T}\left(\vec{r}^{\prime}\right)+\mu_{x c}\right\} \phi_{i}(\vec{r})=\epsilon_{i} \phi_{i}(\vec{r}) .
$$

The electron density at temperature $T, \rho^{T}(\vec{r})$, is given by

$$
\rho^{T}(\vec{r})=\sum_{i} n_{i}^{T}\left|\phi_{i}(\vec{r})\right|^{2}
$$

where $n_{i}^{T}$ is the temperature-dependent occupation number of Fermi-Dirac distribution,

$$
n_{i}^{T}=\frac{1}{1+\mathrm{e}^{\left(\epsilon_{i}-\mu\right) / k_{B} T}}
$$


Here $\epsilon_{i}$ is the energy of electron orbitals, $\mu$ is the chemical potential, and $k_{B} T$ is the temperature in energy units. We note that all the quantities related to the orbitals, $\phi_{i}, \epsilon_{i}$, and $\mu$ depend on the temperature $T$ due to the self-consistency requirement.

For the present purpose, it is essential to use a functional which reproduces both indirect and direct band gaps. The reproduction of the indirect band gap is important to produce correct density of electron-hole pairs for a given electronic temperature. The reproduction of the direct band gap is important for reasonable descriptions of the optical properties. We choose the meta-GGA (generalized-gradient approximation) potential of Tran and Blaha [20] for the exchange-correlation potential, $\mu_{x c}$. The meta-GGA potential depends on the density $\rho^{T}(\vec{r})$, the gradient of the density $\left|\nabla \rho^{T}(\vec{r})\right|$, and the kinetic energy density $\tau^{T}(\vec{r})=$ $\sum_{i} n_{i}^{T}\left|\nabla \phi_{i}(\vec{r})\right|^{2}$. The Tran-Blaha meta-GGA potential is known to resolve to some extent the band gap problem inherent to the local density approximation. It includes a parameter $c$ to which the band gap is sensitive [21]. We treat it empirically, determining $c=1.04$ which reproduces the measured indirect band gap of silicon at $1.17 \mathrm{eV}$. As will be shown later, the optical gap is also found to be described reasonably. The calculated optical gap is about 3.1 $\mathrm{eV}$, in reasonable agreement with the experimental optical gap, $3.4 \mathrm{eV}$ [25].

Practical calculations are achieved as follows. We consider only valence electron orbitals treating electron-ion interaction by a norm-conserving pseudopotential [22, 23]. We use a three-dimensional grid representation to represent orbital wave functions. The cubic unit cell of a side length $a=10.26$ a.u. containing eight silicon atoms is discretized into $20^{3}$ grid points. The $k$-space is also discretized into $32^{3}$ grid points.

Figure 1 shows number density of excited electrons as a function of electron temperature for crystalline silicon. Here, we define the number density of excited electrons $n_{e-h}$ by,

$$
n_{e-h}=\frac{1}{\Omega} \sum_{i=c o n d .} n_{i}^{T},
$$

where the sum is carried out for conduction bands.

As seen from the figure, the number density of excited electrons monotonically increases as the electron temperature increases. At electron temperature of $1.0 \mathrm{eV}$, which corresponds to $11,600 \mathrm{~K}$, the number density of electron-hole pairs is 0.2 per atom, indicating excitations of $5 \%$ of valence electrons. We note that electronic temperatures and number densities of excited electrons shown in Fig. 1 correspond to values of physical interests. It has been often argued that the critical electron density is related to the laser damage threshold. The critical 


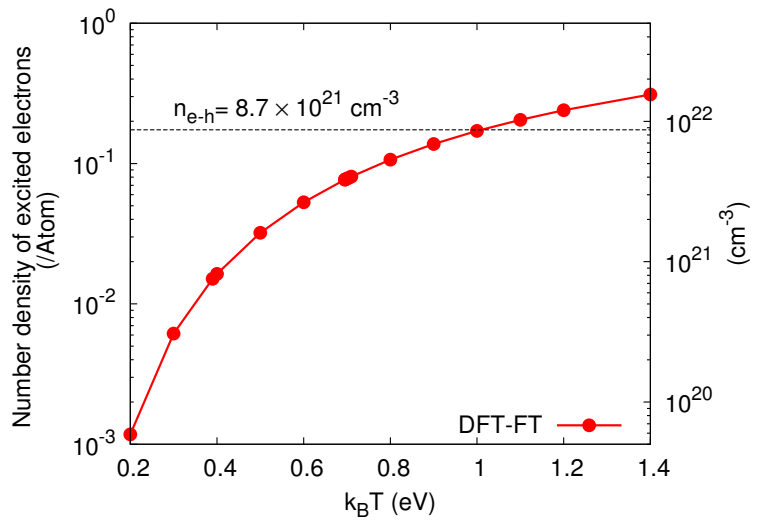

FIG. 1: The number density of electron-hole pairs is shown as a function of electronic temperature in the thermal DFT calculation of crystalline silicon.

electron density is so defined that the plasma frequency of excited carriers coincides with the laser frequency. For Si at $\lambda=625 \mathrm{~nm}$, it is estimated to be $n_{c}=8.7 \times 10^{21} \mathrm{~cm}^{-3}$ [17]. We also note that several experiments have observed laser-excited solids where the number density of excited electrons exceeds $10^{22} \mathrm{~cm}^{-3}$ [17, 24]. In theoretical ab-initio calculations, transition of laser-irradiated silicon into liquid phase has been discussed [28]. In the analysis, initial electronic temperature which is necessary for liquid transition is reported to be 25,000 $\mathrm{K}(2.15 \mathrm{eV})$. In [11], instabilities of phonon modes of silicon are reported following thermal electronic excitations at temperature $1.5 \mathrm{eV}$.

Figure 2 shows occupation distributions at various temperatures, as well as the density of states shown by black solid line. At temperatures around $1 \mathrm{eV}$, we find a substantial excitations of electrons from orbitals within $3 \mathrm{eV}$ below the highest occupied orbital to orbitals within $5 \mathrm{eV}$ above the lowest unoccupied orbitals. From the figure, we find that there is little change in the amount of band gap for wide temperatures. In literatures [26, 27], changes of band gap due to band gap renormalization effect [27] and to a decrease of electron-hole attraction [26] have been investigated. They are originated from screening effects by excited carriers. We consider that these correlation effects are not properly treated in our thermal TDDFT calculation with meta-GGA potential. 


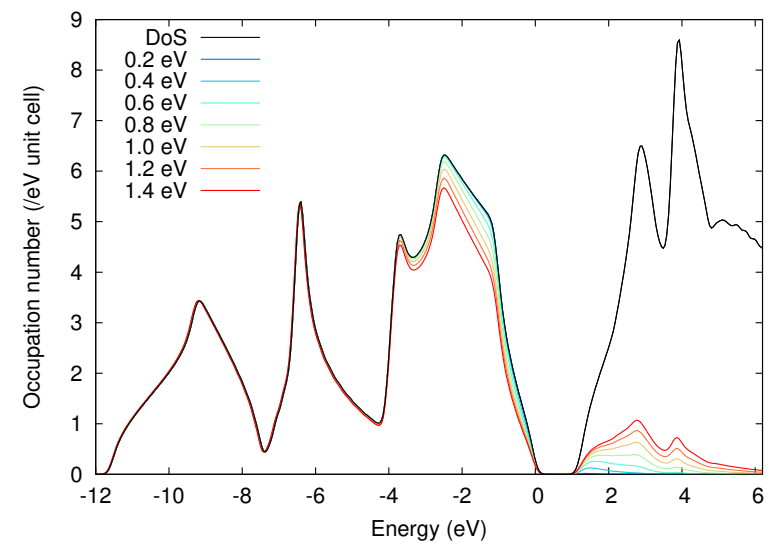

FIG. 2: Occupation number distribution of silicon at various temperatures. The density of states is also shown by black-solid line.

\section{B. Linear response}

We calculate dielectric properties of the medium in the adiabatic TDDFT, using the same Tran-Blaha meta-GGA potential for the response calculation. Numerically, we solve the following time-dependent Kohn-Sham equation in real time to calculate the dielectric property,

$$
i \hbar \frac{\partial}{\partial t} \psi_{i}(\vec{r}, t)=\left\{\frac{1}{2 m_{e}}\left(\vec{p}+\frac{e}{c} \vec{A}(t)\right)^{2}+V_{i o n}+\int d \vec{r}^{\prime} \frac{e^{2}}{\left|\vec{r}-\vec{r}^{\prime}\right|} \rho\left(\vec{r}^{\prime}, t\right)+\mu_{x c}\right\} \psi_{i}(\vec{r}, t) .
$$

The density $\rho(\vec{r}, t)$ is constructed from time-dependent orbitals as $\rho(\vec{r}, t)=\sum_{i} n_{i}^{T}\left|\psi_{i}(\vec{r}, t)\right|^{2}$, using the occupation numbers in the ground states. To explore the dielectric property, we apply a distorting vector potential of step function in time [15, 16].

$$
\vec{A}(t)=\vec{e}_{\beta} A_{0} \theta(t)
$$

where $\vec{e}_{\beta}$ is a unit vector in the $\beta$ direction. We calculate the current flowing within the unit cell from the solution by

$$
\vec{J}(t)=-\frac{e}{\Omega} \sum_{i} n_{i}^{T} \int_{\Omega} d \vec{r} d \vec{r}^{\prime} \psi_{i}^{*}(\vec{r}, t) \vec{v}\left(\vec{r}, \vec{r}^{\prime}\right) \psi_{i}\left(\vec{r}^{\prime}, t\right)
$$

where $\Omega$ is a volume of the unit cell and the velocity operator $\vec{v}(\vec{r}, \vec{r})$ is defined by

$$
\vec{v}\left(\vec{r}, \vec{r}^{\prime}\right)=-\frac{i \hbar}{m_{e}} \vec{\nabla} \delta\left(\vec{r}, \vec{r}^{\prime}\right)+\frac{1}{i \hbar}\left[\vec{r} V_{p s}^{N L}\left(\vec{r}, \vec{r}^{\prime}\right)-V_{p s}^{N L}\left(\vec{r}, \vec{r}^{\prime}\right) \vec{r}^{\prime}\right]
$$


where $V_{p s}^{N L}$ is the nonlocal part of the pseudopotential. The conductivity is calculated from the induced current by

$$
\sigma_{\alpha \beta}(\omega)=-\frac{c}{A_{0}} \int_{0}^{T} d t e^{i \omega t} W(t / T) J_{\alpha}(t)
$$

where $J_{\alpha}(t)$ is the $\alpha$ component of $\vec{J}(t)$, and $T$ is the duration of time evolution. We use the mask function $W(x)$ given by $W(x)=1-3 x^{2}+2 x^{3}$ [13]. The dielectric function is obtained from the conductivity by

$$
\epsilon_{\alpha \beta}(\omega)=\delta_{\alpha \beta}+\frac{4 \pi i \sigma_{\alpha \beta}(\omega)}{\omega}
$$

In silicon, only diagonal element appears in the thermal model, $\epsilon_{\alpha \beta}(\omega)=\delta_{\alpha \beta} \epsilon(\omega)$.

In time evolution calculations, we use the same grid points in the real space and the $k$-space as those in the static calculation. The time propagation is computed using a fourthorder Taylor expansion method [14], with a time step of $\Delta t=0.04$ a.u. The total duration of the time evolution is $T=1,280$ a.u. with the number of time steps $N_{T}=32,000$.

In Fig. 3, we show dielectric functions of silicon at several electron temperatures. In the real part of the dielectric function, all responses at finite temperatures show a strong negative behavior at low frequencies. This Drude-like behavior comes from excited electron-hole pairs. The low energy component of the imaginary part shows absorptive contributions at low frequencies, increasing monotonically as the temperature increases. In our previous study employing numerical pump-probe experiments [6] which catch nonequilibrium distributions of electron-hole pairs, we have observed a similar behavior of Drude-like divergence in the real part. However, the absorptive contribution in the imaginary part was not observed.

A convenient way to exhibit the plasmon contribution to the response is to plot the imaginary part of the inverse dielectric function, $\operatorname{Im} \epsilon^{-1}$. This is shown in Fig. 4 for several temperatures up to $k_{B} T=1.4 \mathrm{eV}$. At the lowest temperature, one sees a very sharp plasmon peak, located at an energy of $\sim 0.4 \mathrm{eV}$. The plasmon excitation energy increases with temperature, due to the increased density of electron-hole pairs. We note that the width of the plasmon also increases with temperature, up to about $k_{B} T \approx 0.6 \mathrm{eV}$. Beyond that, the width does not change very much, up to the maximum temperature considered.

We note that local field corrections are not important in the above results. Namely, results showns above hardly change if we fix the Kohn-Sham Hamiltonian in Eq. (5) to that in the thermal ground state. 


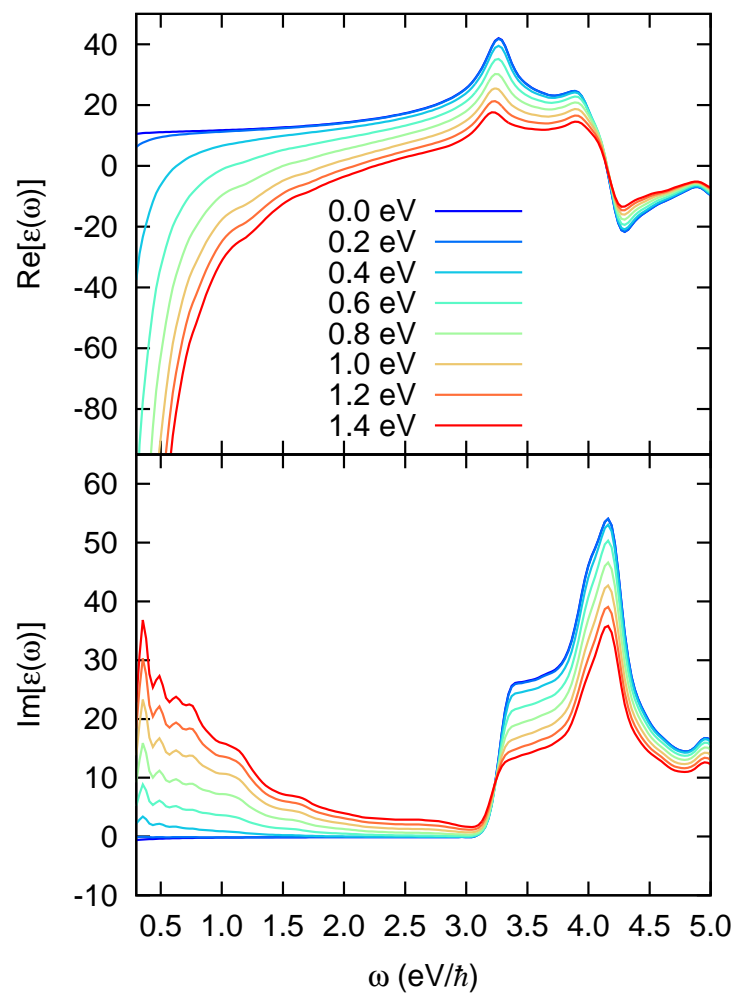

FIG. 3: The dielectric function of silicon in the finite temperature model at several temperatures. Top panel shows the real part of the dielectric function, and the bottom panel shows the imaginary part.

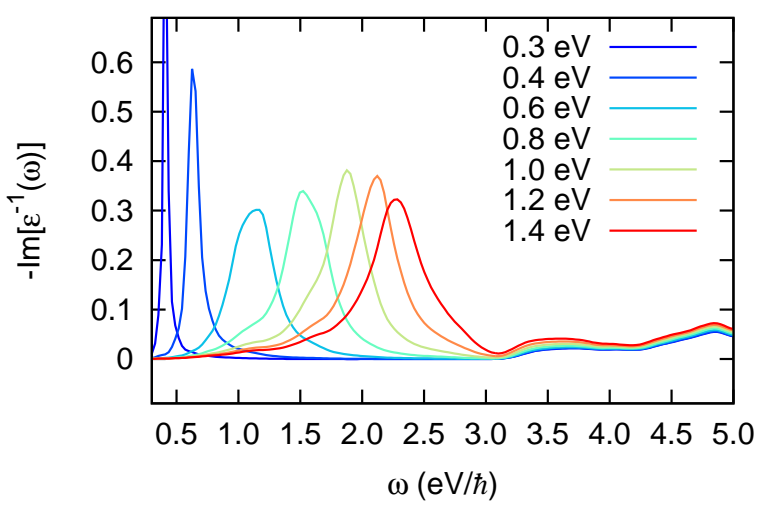

FIG. 4: The imaginary part of the inverse dielectric function for various electronic temperatures. 


\section{COMPARISON WITH FREE-CARRIER MODELS}

The dielectric response of solids excited by intense and ultrashort laser pulses is often modeled by a simplified dielectric function, adding a Drude-like component to the dielectric function in the ground state [18, 19]. In this section, we will show that a model of this kind can reproduce quite well our calculated finite-temperature response.

We consider a model proposed by Sokolowski-Tinten and von der Lind [17], which we shall call the SL model. They consider three physical effects for the dielectric response of laser-excited semiconductor: (i) state and band filling, (ii) renormalization of the band structure, and (iii) the free-carrier response. The SL dielectric function is parameterized as

$$
\begin{aligned}
\epsilon_{S L}(\omega)= & 1+\left[\epsilon_{0}\left(\omega+\Delta E_{\text {gap }}\right)-1\right] \frac{n_{0}-n_{e h}}{n_{0}} \\
& -4 \pi \frac{e^{2} n_{e h}}{m^{*} \omega(\omega+i / \tau)} .
\end{aligned}
$$

Here $\epsilon_{0}(\omega)$ is the dielectric function in the ground state for which we employ the one calculated at zero temperature. $\Delta E_{g a p}$ is the change of the band gap by the laser irradiation for which the calculated shift of the gap energy is used. $n_{e h}$ is the electron-hole pair density for which we use the calculated values. Three other parameters are: the effective mass $m^{*}$; the Drude damping time $\tau$; and the active number of valence electrons $n_{0}$. These are treated as fitting parameters.

The fit is carried out by minimizing the mean square error as given by

$$
I_{\text {error }}=\int_{\omega_{i}}^{\omega_{f}} d \omega\left|\epsilon_{T}^{-1}(\omega)-\epsilon_{S L}^{-1}(\omega)\right|^{2}
$$

where $\epsilon_{T}(\omega)$ is the dielectric function in the thermal model. We take the interval $\hbar \omega_{i}=0.3 \mathrm{eV}$ and $\hbar \omega_{f}=6.0 \mathrm{eV}$. The quality of the fit is shown in Fig. 5 for temperatures of $k_{B} T=1.4$ and $0.4 \mathrm{eV}$ in the thermal model. The fit is very good except for the $\operatorname{Im} \epsilon$ at the lowest frequencies. In particular, the plasmon peak in the inverse dielectric function is very well reproduced.

In Fig. 6, we show the fitted effective mass $m^{*}$ and the collision time $\tau$ as functions of the temperature in the thermal model. The top panel shows that the effective mass $m^{*}$ increases as the temperature increases. We have found a similar behavior in the numerical 

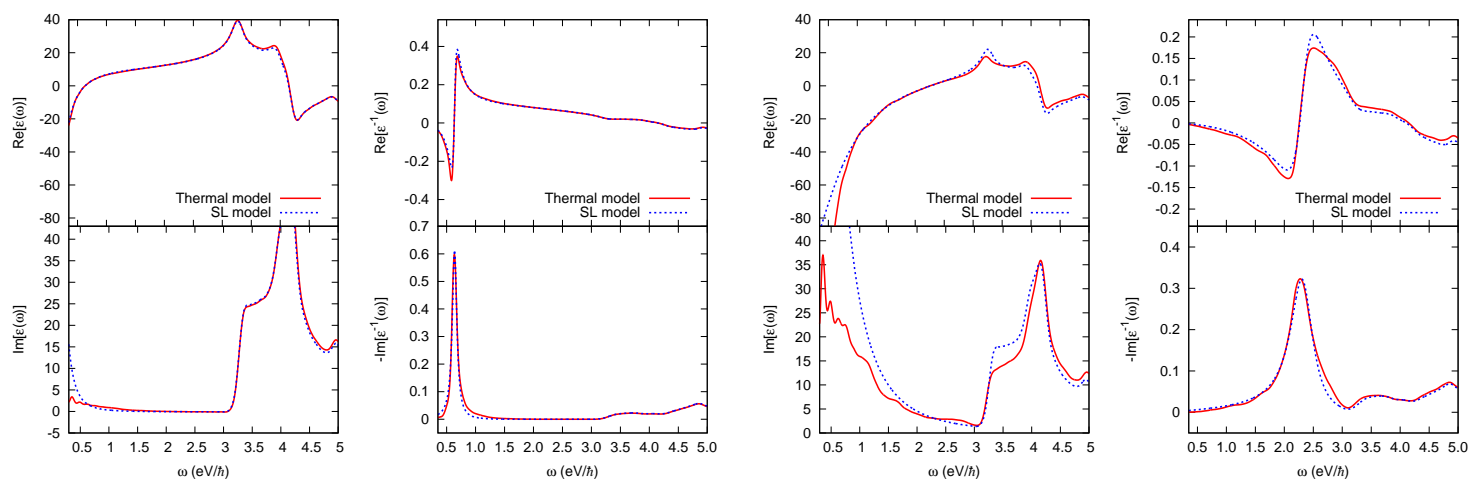

FIG. 5: Comparison of the thermal model and a fit with the SL model. The electronic temperature in the thermal model is $k_{B} T=0.4 \mathrm{eV}$ (left) and $1.4 \mathrm{eV}$ (right).

pump-probe experiments in Ref. [6]. The change of effective mass may be understood by the change of the distribution of the electron-hole pairs in $k$-space.

The bottom panel of Fig. 6 shows that the damping time $\tau$ becomes very small as the electron temperature increases. The value of $\tau$ monotonically decreases and reaches a value of $1.0 \mathrm{fs}$ at $k_{B} T \approx 1.4 \mathrm{eV}$. At first sight this is puzzling, because there are no explicit collision effects in either the TDKS equation or in the thermal model in the adiabatic meta-GGA which we adopted. Since we fix ion positions during time evolution calculations of orbitals, no electron-phonon interactions are taken into account. In spite of them, our plasmon peak has a large damping, corresponding to collision times as short as $1.0 \mathrm{fs}$ in the thermal model. We consider that the damping arises from the elastic scattering of electrons from ionic core potentials. Since the electron-ion interactions constitute periodic potential for electrons, we may equivalently say that the damping is due to the interband transitions of excited carriers. We note that TDDFT treatment of linear response describes the dielectric function of metals fairly well, including the width of plasmon seen in the inverse dielectric function [15].

\section{COMPARISON WITH NUMERICAL PUMP-PROBE EXPERIMENTS}

In the preceding paper [6], we have carried out numerical pump-probe experiments to extract dielectric properties of laser-excited silicon immediately after irradiation by the laser pulse. This method catches fully the nonequilibrium nature of the excited electrons. The 


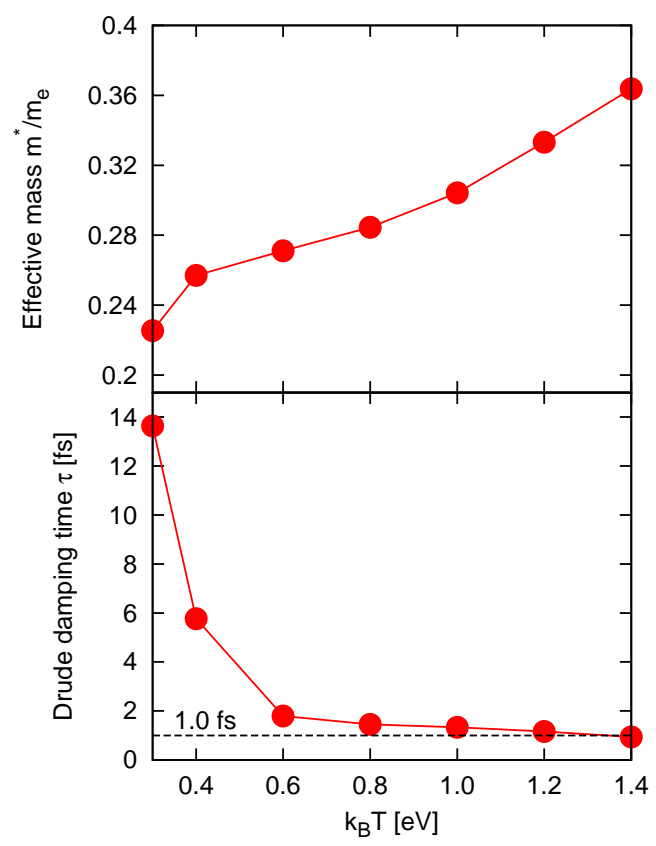

FIG. 6: Parameters of the SL model determined by the fitting procedure to the thermal model. Top panel shows the effective mass $m^{*}$ and the bottom panel shows the Drude damping time $\tau$.

difference between the numerical pump-probe calculations and the present thermal model comes entirely from the different electron-hole distributions in the excited system to be probed. In this subsection, we compare their predicted dielectric functions.

In the numerical pump-probe calculation, we solve the TDKS equation in real time where the electric fields of both pump and probe pulses are included. The pump electric field $E_{P}(t)$ excites electrons and probe electric field $E_{p}(t)$ is used to extract dielectric properties of excited silicon. The dielectric properties are examined from the currents induced by the electric fields. In practice, we performed two calculations. In one calculation, we include both pump and probe electric fields, $E_{P}(t)+E_{p}(t)$, in the TDKS equation. We denote the current in this numerical pump-probe calculation as $J_{P p}(t)$. The other calculation includes only the pump field $E_{P}(t)$ and we denote the current as $J_{P}(t)$. The difference of the currents, $J_{p}(t)=J_{P p}(t)-J_{P}(t)$ brings information of excited silicon. The electric conductivity $\sigma(\omega)$ of excited silicon is given by

$$
\sigma(\omega)=\frac{\int d t J_{p}(t) e^{i \omega t}}{\int d t E_{p}(t) e^{i \omega t}}
$$

and the dielectric function by $\epsilon(\omega)=1+4 \pi i \sigma(\omega) / \omega$. In the numerical pump-probe experi- 


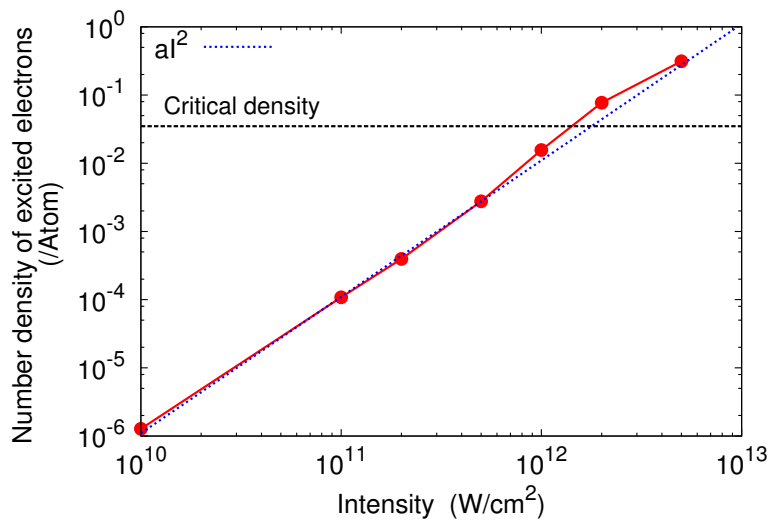

FIG. 7: The number density of electron-hole pairs of the crystalline silicon in the final state following the pulsed excitation as a function of the maximum pump intensity determined as $I=c E_{0}^{2} / 8 \pi$. The critical density is indicated by the horizontal line. The squared intensity line normalized at $10^{10} \mathrm{~W} / \mathrm{cm}^{2}$ is also shown by blue-dotted line. Taken from [6].

ment, we note that the responses are not isotropic but depend on the angle between electric fields of pump and probe fields. We consider two cases: the pump and probe electric fields are parallel and perpendicular to each other.

To compare results of the thermal model with those of the numerical pump-probe experiments, we first need to assume a correspondence between the excited systems that we wish to compare. Since the plasmon characteristics are closely tied to the number of electron-hole pairs, we shall use that measure to make the comparison.

In Ref. [6], we reported calculations solving the TDKS equation with the electric field of the applied laser pulse whose vector potential is given by

$$
A(t)= \begin{cases}-c \frac{E_{0}}{\omega_{P}} \cos (\omega t) \sin ^{2}\left(\pi t / \tau_{L}\right) & \left(0<t<\tau_{L}\right) \\ 0 & \text { (otherwise) }\end{cases}
$$

where $\omega$ and $\tau_{L}$ is the average frequency and the time length of the laser pulse, respectively. $E_{0}$ is the maximum electric field strength in the medium. We denote the maximum intensity of the pulse given by $I=c E_{0}^{2} / 8 \pi$. Using the laser pulse of the frequency $\hbar \omega=1.55 \mathrm{eV}$ and the duration of the pulse $\tau_{L}=18$ fs, the number density of excited electrons is calculated for laser pulses of several intensities. We show the result in Fig. 7 which is taken from [6]. Combining Fig. 7 and Fig. 1, we can relate the laser intensity $I$ and the electronic 

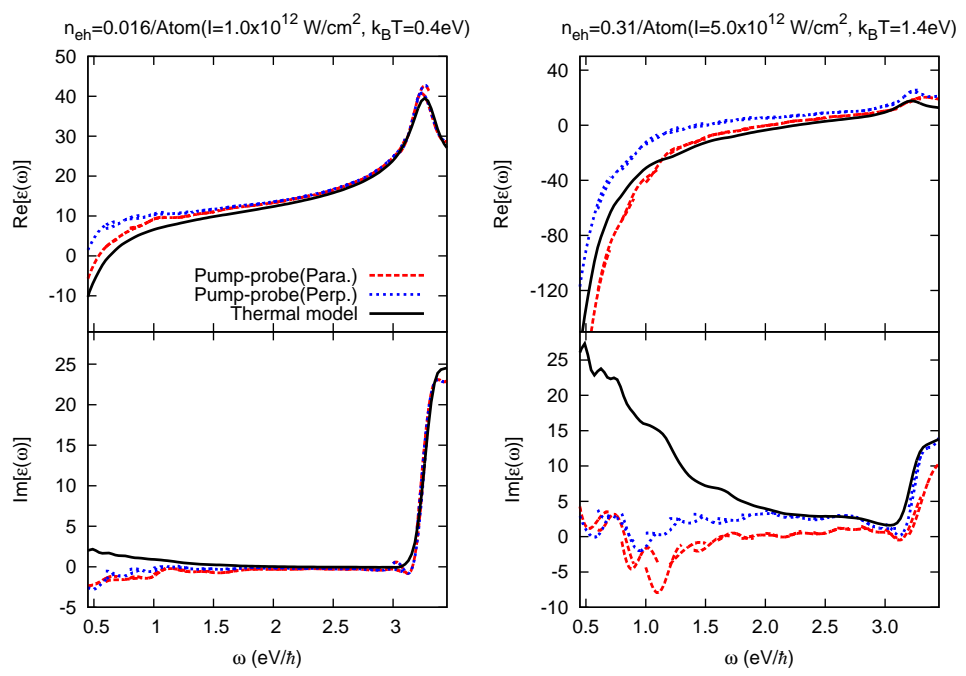

FIG. 8: Comparisons of the dielectric function of the numerical pump-probe calculation [6] and the thermal model. Left-hand panels: $n_{e h}=0.016$ /Atom; right-hand panels: $n_{e h}=0.31$ /Atom.

temperature $k_{B} T$ through the number density of electron-hole pairs $n_{e h}$. For example, in the TDKS calculation using the laser pulse of $I=1.0 \times 10^{12} \mathrm{~W} / \mathrm{cm}^{2}$ excites electron hole pairs of $n_{e h}=0.016 /$ Atom. From Fig. 1, the corresponding temperature is given by $k_{B} T=0.4 \mathrm{eV}$. For the laser pulse of $I=5.0 \times 10^{12} \mathrm{~W} / \mathrm{cm}^{2}$, the density of electron-hole pair is $n_{e h}=0.31 /$ Atom. Corresponding temperature is $k_{B} T=1.4 \mathrm{eV}$. In the following, we use $n_{e h}$ to specify calculations of the finite temperature model and the numerical pump-probe experiments.

We show a comparison of dielectric function by two methods for two cases, $n_{e h}=0.016$ /Atom and $n_{e h}=0.31$ /Atom, in Fig. 8. The black lines show dielectric function of thermal model. The red-dashed line and the blue-dotted line show the results of the numerical pump-probe calculations for probe polarization parallel and perpendicular to the pump, respectively.

As seen from the figure, the real part of the dielectric function of silicon excited by the pump pulse is close to the thermal model for two cases. At lower excitation of $n_{e h}=0.016$ /Atom, the thermal model is close to the pulsed excitation in the parallel probing. At higher excitation of $n_{e h}=0.31$ /Atom, the thermal model is again close to the pulsed excitation in the parallel probing at higher frequencies $(\hbar \omega>1 \mathrm{eV})$ and is between the parallel and perpendicular probings at low frequencies $(\hbar \omega<1 \mathrm{eV})$. The imaginary part of the dielectric function looks rather different. While the thermal model predicts positive imaginary part 
below the band gap, the pulse-excited silicon shows much smaller value, even negative in certain frequencies.

The difference between two calculations comes entirely from different distributions of electron-hole pairs: thermal equilibrium distributions in the thermal model and nonequilibrium distributions in the numerical pump-probe simulation. To clarify the difference, we investigate population distributions in energy and momentum space.

We first denote the orbital index $\{i\}$ in terms of $\{b, \vec{k}\}$, where $b$ indicates bands and $\vec{k}$ indicates the Bloch momentum. Occupation numbers are expressed as $n_{b \vec{k}}^{X}$, where $X=T$ for thermal model and $X=N P P$ for numerical pump-probe simulation. We define the occupation distribution function by

$$
f^{X}(\vec{k}, \epsilon)=\sum_{b} n_{b \vec{k}}^{X} \delta\left(\epsilon-\epsilon_{b \vec{k}}^{X}\right) .
$$

For numerical pump-probe simulation, we define the energy eigenvalue $\epsilon_{b \vec{k}}^{N P P}$ by solving the following Kohn-Sham equation,

$$
\hat{h}_{K S}^{N P P}\left(t_{f}\right) \phi_{b \vec{k}}^{N P P}=\epsilon_{b \vec{k}}^{N P P} \phi_{b \vec{k}}^{N P P},
$$

where $h_{K S}^{N P P}\left(t_{f}\right)$ is the time-dependent Kohn-Sham Hamiltonian at time $t_{f}$ when the laser pulse ended. The occupation number in the numerical pump-probe simulation is defined by

$$
n_{b \vec{k}}^{N P P}=\sum_{b^{\prime} \vec{k}^{\prime}}\left|\left\langle\phi_{b \vec{k}}^{N P P} \mid \psi_{b^{\prime} \vec{k}^{\prime}}^{N P P}\left(t_{f}\right)\right\rangle\right|^{2},
$$

where $\psi_{b \vec{k}}^{N P P}$ is the solution of Eq. (5) at time $t_{f}$.

Using the occupation distribution function, we first calculate the occupation distribution as a function of energy,

$$
D^{X}(\epsilon)=\sum_{\vec{k}}\left\{f^{X}(\vec{k}, \epsilon)-f^{0}(\vec{k}, \epsilon)\right\},
$$

where $f^{0}(\vec{k}, \epsilon)$ is the occupation distribution function in the ground state at zero temperature. The calculated results are shown in Fig. 9 for cases when $n_{e-h}=0.31$ /Atom. Red-solid line shows the distribution of the numerical pump-probe simulation, and greendotted line shows that of the thermal model. We set the highest energy of the valence band to zero. Positive values at positive energy region show distribution of electrons in conduction band, while negative values at negative energy region show the hole distribution in the valence band. 
From the figure, we observe that electrons and holes distribute in wider energy region in the numerical pump-probe simulation than those in the electron thermal model. The decrease of the lower energy electron-hole and the increase of higher energy electron-hole in the numerical pump-probe simulation may cause optical emissions which negatively contribute to the imaginary part of the dielectric function. This explains small or even negative values of the imaginary part of the dielectric function in the numerical pump-probe simulation.

To further clarify the difference in electron-hole distributions, we calculate the distribution in the Bloch momentum space. We note that the Bloch momentum does not correspond to that in the primitive cell since we employ the cubic unit cell containing eight silicon atoms in our calculation. We define the distribution of electrons in the following way:

$$
D_{e}^{X}(\vec{k})=\int_{0}^{\infty} d \epsilon\left\{f^{X}(\vec{k}, \epsilon)-f^{0}(\vec{k}, \epsilon)\right\}
$$

For the distribution of holes, integration is achieved for $\epsilon<0$. We note that there holds $D_{e}^{N P P}(\vec{k})=-D_{h}^{N P P}(\vec{k})$.

Figure 10 (a) shows the distributions of electrons and holes in the thermal model at electron temperature $T=1.4 \mathrm{eV}$, while Figure 10 (b) shows the distribution of electrons in the numerical pump-probe simulation at the pump intensity $I=5.0 \times 10^{12} \mathrm{~W} / \mathrm{cm}^{2}$. The polarization direction of the pump pulse is set parallel to $z$-direction. In both panels, distributions integrated over $k_{y}$ are shown in $k_{x}-k_{z}$ plane. As is evident from panels (a) and (b), there is a large difference in the distribution in momentum space between the thermal model and the numerical pump-probe simulation. In the thermal model, distributions of electrons and holes are different, reflecting the indirect band gap structure. The distribution in the numerical pump-probe simulation shows much more complex, structured, and nonuniform behavior than that in the thermal model, since electrons and holes are in nonequilibrium phase immediately after the laser irradiation. We note that the real parts of the dielectric functions do not show large differences between two calculations (See Fig. 8). This indicates that the real part of dielectric function is sensitive to the number density of excited electrons, not to the detailed distribution of electrons and holes.

We thus conclude that the thermal model describes the real part of the dielectric function quite well, provided the number density of electron-hole pairs is the same. The difference between two calculations comes from the nonthermal distribution of electron-hole pairs in numerical pump-probe simulation. It seems that the difference is more evident for the 


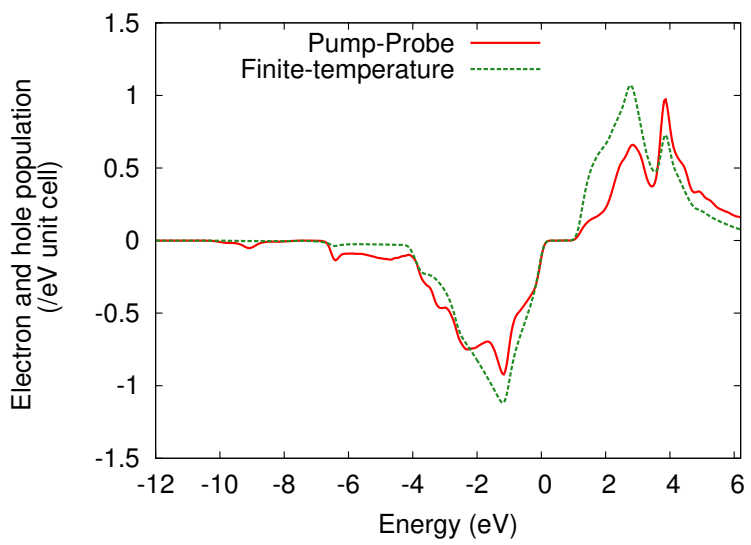

FIG. 9: Population distribution of electrons and holes in laser-excited silicon.

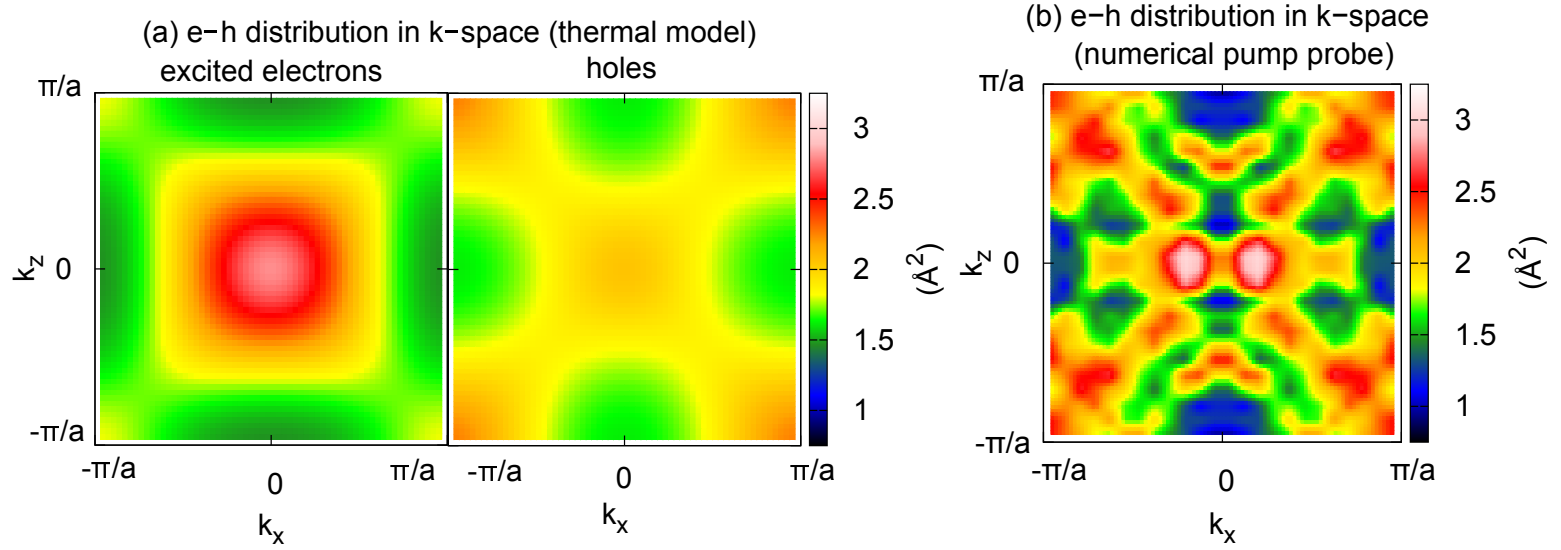

FIG. 10: Population distributions of excited electrons and holes in the Bloch momentum space. The panel (a) shows the excited electron (left hand side) and hole (right hand side) distributions in the thermal model at electron temperature $k_{B} T=1.4 \mathrm{eV}$, while the panel (b) shows the excited electron distribution in the numerical pump-probe method at the pump intensity $I=5.0 \times 10^{12} \mathrm{~W} / \mathrm{cm}^{2}$. The population distributions are shown in the $k_{x^{-}} k_{z}$ plane integrating over $k_{y}$.

imaginary part. A nonequilibrium phase of electronic excitations manifests more sensitively in the imaginary part of the dielectric function.

\section{SUMMARY}

We investigated the change of dielectric response induced by intense and ultrashort laser pulses by a thermal model, assuming electronic equilibrium. This description is expected to apply to excited matter after a few tens of femtosecond following the laser irradiation. 
We first solved the static Kohn-Sham equation with finite temperature Fermi-Dirac function occupation factors. Its dielectric response was then computed by applying the linear response theory using the real-time method.

The calculated thermal dielectric function is characterized by the strong negative behavior in the real part at low frequencies caused by excited electron-hole pairs. The imaginary part shows absorptive contributions at low frequencies, increasing monotonically as the temperature increases. Plotting it in the inverse dielectric function, a sharp plasmon feature manifests clearly. The frequency of the plasmon increases monotonically with temperature due to the increased density of electron-hole pairs. The width also increases at low temperature region, then becomes almost constant.

The thermal dielectric function is compared with a simple Drude model of free-electron dynamics, embedded in the dielectric medium corresponding to the ground state. There are three basic parameters determining the electron-hole plasma properties, namely the density of electron-hole pairs, their effective mass $m^{*}$, and the collision time $\tau$. The density of electron-hole pairs is known from the thermal ground state calculation, but the other quantities are fit. We find the collision time of as short as $1.0 \mathrm{fs}$ gives reasonable fit. This short value for the collision time is unexpected, since there are no explicit collision terms in the time-dependent Kohn-Sham equation that we solve. We consider the short collision time comes from the elastic scattering of electrons from atoms.

We also compared the thermal dielectric function with that derived from numerical pumpprobe calculation in which electronic response is derived from time evolution of Kohn-Sham orbitals under electric fields of both pump and probe pulses. The numerical pump-probe simulation describes the response of excited matter in the nonequilibrium state reached just after the pulse has been applied. We find the real part of the dielectric function shows reasonable correspondence if we compare them at the same number density of electronhole pairs. However, the imaginary part shows marked difference. The thermal dielectric function shows positive imaginary part, while the numerical pump-probe calculation gives small contribution in the imaginary part, even negative contribution.

The above difference comes from the distributions of electrons and holes. To clarify the origin of the difference, we investigated the distribution in energy and momentum space. From the population distribution in energy domain, we found that electrons and holes distribute in wider energy region in the numerical pump-probe simulation than those in the 
thermal model. The decrease of the lower energy electron-hole and the increase of higher energy electron-hole in the numerical pump-probe simulation may cause optical emission which negatively contribute to the imaginary part of the dielectric function. This explains small or even negative values of the imaginary part of the dielectric function in the numerical pump-probe simulation. From the population distribution in Bloch momentum, we found large differences between the thermal model and the pump-probe simulation. The distribution in the numerical pump-probe simulation is much structured and nonuniform compared with the thermal case, reflecting nonequilibrium phase immediately after the end of the incident pulse.

In spite of the large difference of the electron-hole distributions between the thermal model and the numerical pump-probe simulation, the real parts of the dielectric functions are qualitatively similar. Moreover, the real parts of the dielectric functions in both cases can be well described by the Drude model. The real part of the dielectric is well described by the Drude model using only the number density of excited-electrons and the effective mass. Therefore, we may validate the estimation of the number density of excited-electrons in laser-excited solids using the Drude model for both non-equilibrium and thermal phases, based on the microscopic treatment of the quantum electron dynamics.

\section{Acknowledgments}

We thank G.F. Bertsch for discussions and suggestions. This work is supported by the Grants-in-Aid for Scientific Research No. 23340113, No. 23104503, No. 21340073, and No. 21740303. The numerical calculations were performed on the supercomputer at the Institute of Solid State Physics, University of Tokyo, and T2K-Tsukuba at the Center for Computational Sciences, University of Tsukuba.

[1] M.D. Perry, B.C. Stuart, P.S. Banks, M.D. Feit, V. Yanovsky, A.M. Rubenchik, J. Appl. Phys. 85, 6803 (1999).

[2] T. Brabec, F. Krausz, Rev. Mod. Phys. 72, 545 (2000).

[3] A. Couairon and A. Mysyrowicz, Phys. Rep. 441, 47 (2007).

[4] E.G. Gamaly, Phys. Rep. 508, 91 (2011). 
[5] P. Balling and J. Schou, Rep. Prog. Phys. 76, 036502 (2013).

[6] S.A. Sato, K. Yabana, Y. Shinohara, T. Otobe, and G.F. Bertsch, Phys. Rev. B89, 064304 (2014).

[7] B.Y. Mueller and B. Rethfeld, Phys. Rev. B87, 035139 (2013).

[8] N.D. Mermin, Phys. Rev. 137, A1441 (1965).

[9] S. Pittalis, C.R. Proetto, A. Floris, A. Sanna, C. Bersier, K. Burke, and E.K.U. Gross, Phys. Rev. Lett. 107, 163001 (2011).

[10] K. Shiratori and K. Nobusada, J. Phys. Chem. A112, 10681 (2008).

[11] V. Recoules, J. Clérouin, G. Zérah, P.M. Anglade, and S. Mazevet, Phys. Rev. Lett. 96, $055503(2006)$.

[12] S.M. Vinko et.al, Phys. Rev. Lett. 104, 225001 (2010).

[13] K. Yabana, T. Nakatsukasa, J.-I. Iwata, and G.F. Bertsch, phys. stat. sol. (b)243, 1121 (2006).

[14] K. Yabana and G.F. Bertsch, Phys. Rev. B54, 4484 (1996).

[15] G.F. Bertsch, J.-I. Iwata, A. Rubio, and K. Yabana, Phys. Rev. B 627998 (2000).

[16] K. Yabana, T. Sugiyama, Y. Shinohara, T. Otobe, G.F. Bertsch, Phys. Rev. B 85045134 (2012).

[17] K. Sokolowski-Tinten and D. von der Linde, Phys. Rev. B 61, 2643 (2000).

[18] N. Medvedev and B. Rethfeld, J. Appl. Phys. 108, 103112 (2010)

[19] B. Rethfeld, O. Brenk, N. Medvedev, H. Krutsch, D.H.H. Hoffmann, Appl. Phys. A101 19, (2010).

[20] F. Tran and P. Blaha, Phys. Rev. Lett. 102, 226401 (2009).

[21] D. Koller, F. Tran, and P. Blaha, Phys. Rev. B85, 155109 (2012).

[22] N. Troullier and J.L. Martins, Phys. Rev. B43, 1993 (1991).

[23] L. Kleinman and D. M. Bylander, Phys. Rev. Lett. 48, 1425 (1982).

[24] D. Hulin, M. Combescot, J. Bok, A. Migus, J. Y. Vinet, and A. Antonetti, Phys. Rev. Lett. 52, 1998 (1984).

[25] Ricardo R. L. Zucca and Y. R. Shen, Phys. Rev. B 1, 2668 (1970).

[26] Lorin X. Benedict, Phys. Rev. B 63, 075202 (2001).

[27] Sergey V. Faleev, Mark van Schilfgaarde, Takao Kotani, François Léonard, and Michael P. Desjarlais, Phys. Rev. B 74, 033101 (2006).

[28] P.L. Silvestrelli, A. Alavi, M. Parrinello, D. Frenkel, Phys. Rev. Lett. 77, 3149 (1996). 